\title{
Fluoride concentration and stability in commonly used dentifrices in Sri Lanka
}

\author{
Niroshani Surangika Soysa', Jaime Aparecido Cury², \\ Chrisman Neil Roshan Alexander Alles ${ }^{3}$
}

1 Division of Pharmacology, Department of Oral Medicine and Periodontology, Faculty of Dental Sciences, University of Peradeniya, Sri Lanka;

2 Oral Biochemistry, Piracicaba Dental School, University of Campinas, Brazil;

${ }^{3}$ Department of Biochemistry, Faculty of Medicine, University of Peradeniya, Sri Lanka.

Corresponding author: Niroshani S. Soysa, BDS, PhD Pharmacology, Dept. of Oral Medicine and Periodontology, Faculty of Dental Sciences University of Peradeniya, Sri Lanka. E-mail:niroshanis@pdn.ac.lk, hnsnit@yahoo.com, nickysoysa@gmail.com Tel: 0094- 81-2397470

Received: June 14, 2018

Accepted: October 11, 2018
Aim: According to the best scientific evidence, a dentifrice should contain 1000 ppm $\mathrm{F}$ or above but to guarantee the anti-caries efficacy this concentration should be chemically soluble and remain stable until the dentifrice expiration date. This stability depends on the type of abrasive and fluoride salt used in the formulation. Nine brands of dentifrices purchased in the Sri Lankan market were evaluated. Methods: The total fluoride (TF = soluble + insoluble) and total soluble fluoride (TSF = ion MFP + ion F-) concentrations were determined in fresh samples and after storage at room temperature and after accelerated aging. The analyses were made with an ion specific electrode using a validated analytical method. Three dentifrices were formulated with $\mathrm{MFP} / \mathrm{CaCO}_{3}$, two with MFP/Silica, two with NaF/Silica and two were not fluoridated. Results: The TF concentration found was very close to the concentration declared by the manufacturer. However, even in the fresh sample of one dentifrice formulated with $\mathrm{MFP} / \mathrm{CaCO}_{3}$, there was insoluble fluoride (22\%). The TSF concentration in all $\mathrm{MFP} / \mathrm{CaCO}_{3}$-based dentifrices decreased with storage while insoluble fluoride increased. The dentifrices formulated with MFP/Silica and NaF/Silica showed chemical stability. Conclusion: This is the first study done about the chemical quality of fluoride dentifrices marketed in Sri Lanka and the findings are an alert for a surveillance system in the country.

Keywords: Fluorides. Dental caries. Dentifrices. 


\section{Introduction}

Water fluoridation and dentifrices are considered to be the most effective ways of fluoride $(F)$ use in controlling caries ${ }^{1}$. Although $F$ does not have a direct effect on the etiological factors responsible for dental caries, it is very effective in reducing the progression of caries lesions and dentifrices are considered to be the most rational way of $F$ use because when dental plaque is disrupted by toothbrushing, fluoride is delivered to the oral cavity at the same time to interfere with the caries process ${ }^{1,2}$.

The regular use of fluoridated toothpaste is responsible for the marked reduction in dental caries prevalence in both the developed and developing countries ${ }^{3-5}$. However, F should be chemically free and in the soluble form in the dentifrice formulation, either as ion fluoride $\left(\mathrm{F}^{-}\right)$from the NaF toothpastes or as ion MFP from sodium monofluorophosphate dentifrices $\left(\mathrm{Na}_{2} \mathrm{PO}_{3} \mathrm{~F}\right)$ to exert its mechanism of action, acting on the tooth/plaque interface to enhance remineralization and reduce the enamel solubility by reducing demineralization ${ }^{6}$. The availability of the free soluble form of $F$ depends on the chemical compatibility between the type of salt $\mathrm{F}$ mentioned above and the abrasive used. When the dentifrice contains calcium $\left(\mathrm{Ca}^{2+}\right)$ in the abrasive $\left(\mathrm{CaCO}_{3}\right.$ or $\left.\mathrm{CaHPO}_{4} \cdot 2 \mathrm{H}_{2} \mathrm{O}\right)$, it must be formulated with $\mathrm{Na}_{2} \mathrm{FPO}_{3}$ In most developing countries, dentifrices are formulated with $\mathrm{MFP} / \mathrm{CaCO}_{3}$. Though MFP is more compatible with $\mathrm{CaCO}_{3}$ or $\mathrm{CaHPO}_{4} \cdot 2 \mathrm{H}_{2} \mathrm{O}$, a part of total $\mathrm{F}$ in these formulations is insoluble because during storage MFP is hydrolyzed, releasing $\mathrm{F}$ ion that react with $\mathrm{Ca}^{2+}$ and form salts of low solubility such as $\mathrm{CaF}_{2}{ }_{2}$. On the other hand, due to the incompatibility of $\mathrm{NaF}, \mathrm{SnF}_{2}$ and even amine fluorides with calcium-based abrasives, silica $\left(\mathrm{SiO}_{2}\right)$ particles are used as abrasives in these formulations where $\mathrm{F}$ is chemically soluble and readily bioavailable. Therefore, it is believed that at least 1000 ppm of soluble $F$ is necessary for a dentifrice to have its anti-caries effect ${ }^{8}$. The total $F$ concentration (TF) does not clearly give an idea about how much $\mathrm{F}$ is soluble in a dentifrice. Moreover, aging reduces the soluble fluoride concentration in a toothpaste ${ }^{9-11}$.

Brazil is a good example for a developing country which has achieved a marked reduction in the prevalence of caries by introducing the topical application of $F$ by using dentifrices. The current legislation of Brazil sets the maximum $F$ concentration at 1500 ppm F, but does not specify whether the available $F$ should be either Total $F$ (TF) or Total Soluble F (TSF). A recent study done in Brazil revealed that though TF declared on the labels were in accordance with the mentioned amount, the concentration of TSF was $21 \%$ less than the TF declared, suggesting that the reduction in F is due to the aging of the toothpaste since the abrasive used in a majority of those formulations is $\mathrm{CaCO}_{3}$. Nevertheless, the TSF concentration was above $1000 \mathrm{ppm} F$ which is the minimum concentration necessary for an anti-caries effect? .

Sri Lanka introduced F dentifrices in 1983 and after that three national oral surveys have been carried out in Sri Lanka. According to the surveys done in 1994/199512 and $2002 / 2003^{13}$, though there was a reduction in caries prevalence in the 12 year, 15 year and $35-44$ year groups, it was not satisfactory as by 2002/2003, 39.2\%, 52.3\% and $89.8 \%$, respectively were having caries ${ }^{13}$. There could be many reasons for that and 
one important factor is that the F concentration recommended by the Sri Lanka Standards is $650-1100$ ppm $F$ which is below the minimum recommended anti-caries concentration of 1000 ppm F. In addition, the common usage of $\mathrm{CaCO}_{3}$ as the abrasive might cause the aging of toothpastes, giving rise to sub-therapeutic concentrations of $F$, undermining the beneficial anti-caries effect of topical $F$.

Not a single study has been done either to establish the availability and stability of $F$ in toothpastes or the availability of TSF in the MFP/CaCO${ }_{3}$-based formulations during storage. Therefore, this is the first study carried out in Sri Lanka which aims to analyze the concentration of TF and TSF in the dentifrices available in the Sri Lankan market and to analyze how their aging affects the TSF with time.

\section{Material and Methods}

\section{Samples and experimental design}

For the current study, nine different types of locally manufactured and imported dentifrices (toothpastes) available in the Sri Lankan market were purchased off the shelf from supermarkets in the Kandy District, Sri Lanka. Supermarkets were selected due to their high turnover rates of toothpastes and other facilities. All the information about the total F concentration, abrasive added, manufacturing date, expiry date and other relevant information were obtained from the packaging. Toothpastes were stored at a residential ambient temperature and the analyses were carried out at the time of acquisition and after 6 months, 12 months (by accelerated aging) and 24 months (natural aging). Ethical approval was obtained from the Ethics Review Committee of the Faculty of Dental Sciences, University of Peradeniya, Sri Lanka.

\section{Preparation and determination of fluoride concentration}

The fluoride concentration in the dentifrices was determined as described elsewhere ${ }^{14}$ and is briefly as follows (Figure 1). An amount of $90-110 \mathrm{mg}$ of dentifrice was weighed, homogenized in $10 \mathrm{ml}$ of deionized water and duplicates of $0.25 \mathrm{ml}$ of suspension were transferred to assay tubes for TF (the sum of soluble and insoluble fluoride) analysis. The suspension was centrifuged (5,000 g, $10 \mathrm{~min}$, room temperature) to remove insoluble fluoride bound to the abrasive. Duplicates of $0.25 \mathrm{ml}$ of the supernatant were transferred to assay tubes to determine the TSF (sum of F as F ion and MFP ion) concentration. To all the TF and TSF tubes, $0.25 \mathrm{ml}$ of $2.0 \mathrm{M} \mathrm{HCl}$ was added, and after $1 \mathrm{hr}$ at $45^{\circ} \mathrm{C}$, the samples were neutralized with $0.50 \mathrm{ml}$ of $1 \mathrm{M} \mathrm{NaOH}$ and buffered with $1 \mathrm{ml}$ of TISAB II ( $1 \mathrm{M}$ acetate buffer, $\mathrm{pH} 5.0$, containing $1 \mathrm{M} \mathrm{NaCl}$ and $0.4 \%$ CDTA). The analyses were carried out using an ion-specific electrode.

\section{Accelerated aging of dentifrices}

Accelerated aging of dentifrices was performed as described elsewhere ${ }^{15}$. Before analyzing, non-fluoridated toothpastes and NaF-based toothpastes were aged for 8 days at $45^{\circ} \mathrm{C}$ and MFP-based toothpastes were aged for 16 days at $55^{\circ} \mathrm{C}$. Fluoride analysis was performed as mentioned above and as described elsewhere ${ }^{14}$. 


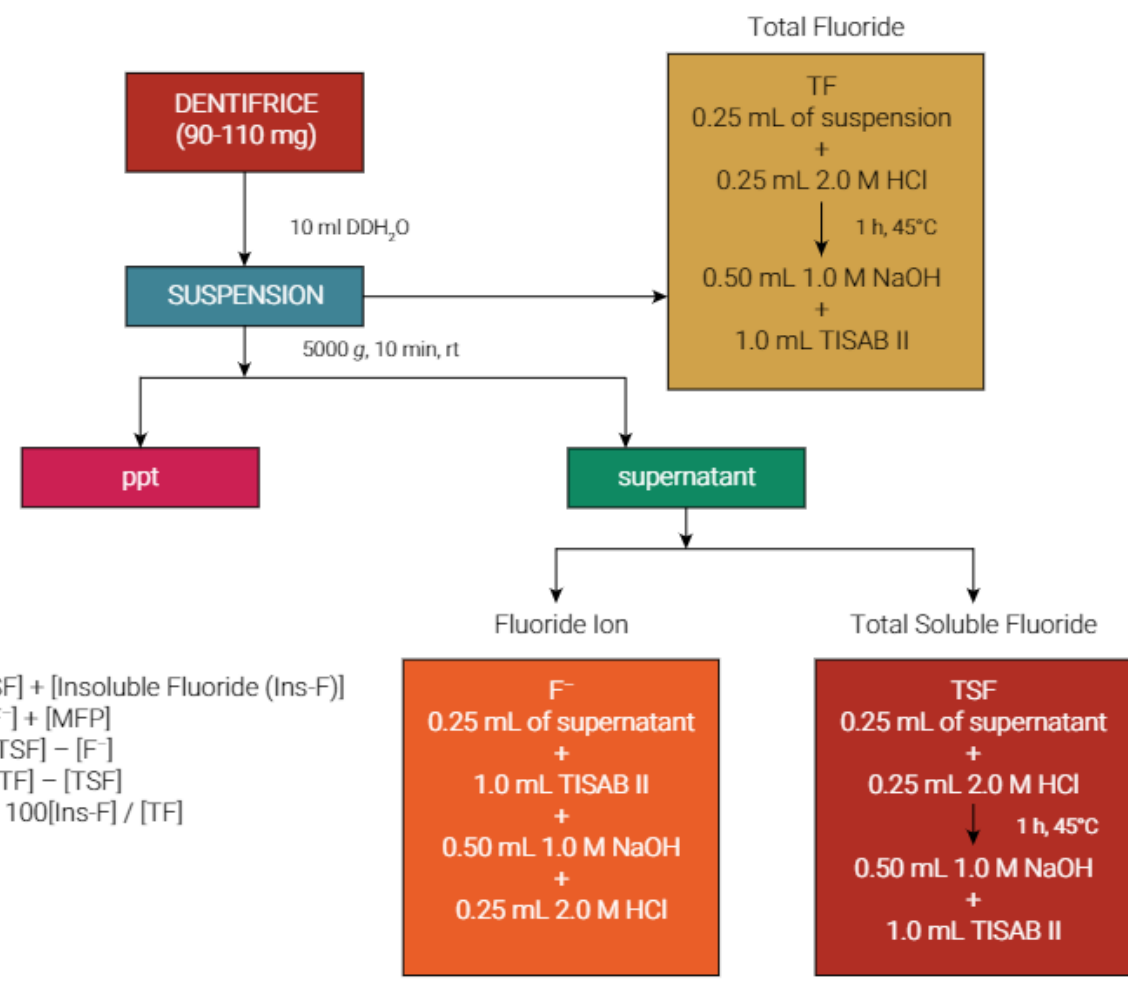

Figure 1. Schematic diagram of the protocol of analysis of total fluoride (TF), total soluble fluoride TSF), ion fluoride (F) and the calculations of F as MFP, and the \% of insoluble fluoride in toothpastes.

\section{Fluoride determination}

The fluoride concentrations in the dentifrices was determined as described elsewhere ${ }^{14}$. In brief, a specific electrode for fluoride Thermo Orion 96-09 coupled to the ion analyzer, Thermo Orion Star A214 (Orion Research Inc., Boston, USA) was used for all analyses. Calibration was performed previously with standard $\mathrm{F}$ solutions containing a final concentration of $0.0625,0.125,0.25,0.5,1.0,2.0,4.0 \mathrm{ppm}(\mathrm{mg} \mathrm{F} / \mathrm{ml}$,) prepared in $0.25 \mathrm{M} \mathrm{HCL}, 0.25 \mathrm{M} \mathrm{NaOH}$ and TISAB II $50 \%(\mathrm{~V} / \mathrm{V})$.

For each sample, a linear equation correlating with the logarithm of $\mathrm{F}$ concentration in standards and $\mathrm{mV}$ was calculated using the software, EXCEL (Microsoft) and was used to determine the $\mathrm{F}$ concentration in each dentifrice which was then expressed as ppm (mg F/K). The average coefficient of variation within each independent analysis was lower than $3 \%$.

\section{Statistical analysis}

The mean concentration of fluoride and the standard deviation (SD) were calculated for each toothpaste using Microsoft Excel.

\section{Results}

In the current study, we have used nine commercially available toothpastes to analyze the changes in $\mathrm{F}$ concentrations with time (aging). All the toothpastes were coded 
from A to I. Out of the nine toothpastes, three fluoridated toothpastes, B, F, and G, have been manufactured in Sri Lanka (SL) while A, C, D and E have been imported. $\mathrm{H}$ and I were non-fluoridated, herbal toothpastes manufactured in SL. D and E were the ones which are usually prescribed for tooth sensitivity (Table 1). Out of the five monofluorophsphate (MFP)-based formulations, three of the locally manufactured fluoridated toothpastes contained calcium carbonate $\left(\mathrm{CaCO}_{3}\right)$ as the abrasive while A and D contained hydrated silica. A and $\mathrm{E}$ had declared having a fluoride concentration of 1000 ppm $F$ while $B$ and $C$ gave a range between 650-1150 ppm F. F and $G$ had a fluoride concentration in the range of 850-1150 ppm F (Table 1).

Table 2 shows the concentrations of total fluoride (TF), total soluble fluoride (TSF) and insoluble fluoride (IF) at the time of acquisition of the toothpastes. Total fluoride represents all forms of available fluoride (soluble and insoluble); total soluble fluoride is the sum of ionizable fluoride such as MFP and ionic fluoride $\left(\mathrm{F}^{-}\right)$. Insoluble fluoride represents the percentage of fluoride that is bound to the abrasive system and which is not available for the control of caries. Two of the toothpaste formulations, $\mathrm{H}$ and I were non-fluoridated toothpastes (Table 1). Though all the fluoridated toothpastes contained at least 900 ppm F, which is within the recommended concentrations of the Sri Lankan Standards, (i.e. 650-1100 ppm), only two (B and G) had TSF concentrations above 1000 ppm F. Interestingly, one locally manufactured toothpaste (B) had more TF (1584 ppm F) than what was declared on the label (Table 1). For the $\mathrm{F}$ to exert its effect it should be in the soluble form. The percentage of insoluble fluoride was lower than $1 \%$ in all the formulations except in one (B) which had

Table 1. Codes and label information of the toothpastes analyzed (MFP* = Sodium monoflurophosphate; $\mathrm{NaF}^{\star \star}=$ Sodium fluoride.

\begin{tabular}{|c|c|c|c|c|c|}
\hline \multirow[b]{2}{*}{ Code } & \multirow[b]{2}{*}{ Description of dentifrices } & \multicolumn{4}{|c|}{ Label information } \\
\hline & & $\begin{array}{l}\text { Type of } \\
\text { Abrasive }\end{array}$ & $\begin{array}{l}\text { Type } \\
\text { of F }\end{array}$ & $\begin{array}{c}\mathrm{ppm} F \\
(\mathrm{mg} \mathrm{F} / \mathrm{kg})\end{array}$ & $\begin{array}{l}\text { Lot number and } \\
\text { manufacture } \\
\text { date }\end{array}$ \\
\hline A & $\begin{array}{l}\text { Colgate Max fresh } \\
\text { Imported }\end{array}$ & $\begin{array}{l}\text { Hydrated } \\
\text { silica }\end{array}$ & MFP* & 1000 & B1903/14 \\
\hline B & $\begin{array}{c}\text { Clogard } \\
\text { Locally manufactured }\end{array}$ & $\mathrm{CaCO}_{3}$ & MFP & $650-1150$ & $\begin{array}{c}\mathrm{G} 142 \mathrm{H} \\
12 / 07 / 14\end{array}$ \\
\hline $\mathrm{C}$ & $\begin{array}{l}\text { Close up deep action } \\
\text { Imported }\end{array}$ & $\begin{array}{l}\text { Hydrated } \\
\text { silica }\end{array}$ & $\mathrm{NaF}^{\star *}$ & $650-1150$ & $\begin{array}{c}\mathrm{BC} 01: 06 \\
11 / 07 / 14\end{array}$ \\
\hline D & $\begin{array}{c}\text { RA Thermoseal } \\
\text { Imported. Prescribed for sensitive teeth }\end{array}$ & $\begin{array}{l}\text { Hydrated } \\
\text { silica }\end{array}$ & MFP & 917 & $\begin{array}{l}30416 \\
10 / 13\end{array}$ \\
\hline$E$ & $\begin{array}{l}\text { Sensodyne Fresh Mint } \\
\text { Imported. Prescribed for sensitive teeth }\end{array}$ & $\begin{array}{l}\text { Hydrated } \\
\text { silica }\end{array}$ & $\mathrm{NaF}$ & 1000 & $\begin{array}{c}100114 \\
01 / 14\end{array}$ \\
\hline $\mathrm{F}$ & $\begin{array}{c}\text { Signal Herbal } \\
\text { Locally manufactured }\end{array}$ & $\mathrm{CaCO}_{3}$ & MFP & $850-1150$ & $\begin{array}{c}\mathrm{BC} 06: 19 \\
18 / 07 / 14\end{array}$ \\
\hline G & $\begin{array}{l}\text { Signal strong teeth } \\
\text { Locally manufactured }\end{array}$ & $\mathrm{CaCO}_{3}$ & MFP & $850-1150$ & $\begin{array}{c}\text { BC18:03 } \\
13 / 06 / 14\end{array}$ \\
\hline $\mathrm{H}$ & $\begin{array}{l}\text { Sudantha } \\
\text { Locally manufactured non-fluoridated } \\
\text { tooth paste }\end{array}$ & \multirow{2}{*}{\multicolumn{3}{|c|}{ Non-fluoride }} & $\begin{array}{c}\text { D538 } \\
14 / 07 / 14\end{array}$ \\
\hline 1 & $\begin{array}{c}\text { Supirivicky } \\
\text { Locally manufactured non-fluoridated } \\
\text { tooth paste }\end{array}$ & & & & $\begin{array}{c}629 D \\
24 / 04 / 14\end{array}$ \\
\hline
\end{tabular}


about $22.4 \%$ of insoluble fluoride (Table 2). Non-fluoridated formulations as well as NaF-based formulations ( $\mathrm{C}$ and $\mathrm{E}$ ) were not affected by six months of natural aging as expected (Table 3). The same was observed with the MFP-based formulations which did not contain calcium (A and D). Though the total soluble concentration of $B$ seemed to have decreased, 6 months of natural aging had not affected the TSF in toothpastes of $\mathrm{F}$ and $\mathrm{G}$ markedly (Table 3 ).

The non-fluoridated formulations as well as NaF-based formulations were not affected by accelerated aging which mimicked the one year of natural aging as expected (Table 4). Furthermore, the MFP-based formulations that did not contain calcium (A and D) were not affected by accelerated aging. However, calcium contain-

Table 2. Concentrations of total fluoride (TF), total soluble fluoride (TSF as ion MFP and ion F) and the percentage of insoluble fluoride found in the fresh samples (at time of acquisition) of the toothpastes. Data are expressed as the mean \pm SD. Samples were analyzed in triplicate and the representative of the two independent experiments is shown.

\begin{tabular}{lcccc}
\hline \multirow{2}{*}{ Dentifrices } & Total Fluoride & \multicolumn{2}{c}{ Total soluble fluoride (TSF) } & \% Insoluble F \\
\cline { 3 - 4 } & $(\mathrm{TF})$ & MFP & lonic $F$ & \\
\hline $\mathrm{A}$ & $928.5 \pm 30.6$ & $902.4 \pm 8.9$ & $49.5 \pm 4.3$ & $<0.5$ \\
\hline $\mathrm{B}$ & $1584.9 \pm 18.8$ & $985.4 \pm 4.8$ & $244.2 \pm 9.9$ & $22.4 \pm 0.6$ \\
\hline $\mathrm{C}$ & $934.3 \pm 25.9$ & - & $915.1 \pm 3.0$ & - \\
\hline $\mathrm{D}$ & $892.3 \pm 22.7$ & $866.3 \pm 34.1$ & $51.5 \pm 3.0$ & $<0.5$ \\
\hline $\mathrm{E}$ & $990.4 \pm 6.0$ & - & $981.7 \pm 47.4$ & - \\
\hline $\mathrm{F}$ & $973.9 \pm 42.8$ & $806.9 \pm 33.4$ & $179.3 \pm 2.3$ & $<0.5$ \\
\hline $\mathrm{G}$ & $1065.8 \pm 13.9$ & $826.5 \pm 0.02$ & $232.5 \pm 8.0$ & $0.6 \pm 0.6$ \\
\hline $\mathrm{H}$ & $8.8 \pm 0.6$ & - & $6.8 \pm 1.1$ & - \\
\hline I & $9.7 \pm 0.3$ & - & $7.3 \pm 1.4$ & - \\
\hline
\end{tabular}

Table 3. Concentrations of total fluoride (TF), total soluble fluoride (TSF as ion MFP and ion F) and the percentage of insoluble fluoride found after 6 months of aging at room temperature.\#- this was from a different batch which was manufactured 3 months prior to the previous one. \$ and $\$ \$$ happened to be from different batches which were manufactured within one month of the previous ones. Data are expressed as the mean \pm SD. Samples were analyzed in triplicate and the representative of the two independent experiments is shown.

\begin{tabular}{|c|c|c|c|c|}
\hline \multirow{2}{*}{ Dentifrices } & \multirow{2}{*}{$\begin{array}{l}\text { Total Fluoride } \\
\text { (TF) }\end{array}$} & \multicolumn{2}{|c|}{ Total soluble fluoride (TSF) } & \multirow{2}{*}{$\%$ Insoluble F } \\
\hline & & MFP* & Ionic F & \\
\hline A & $958.2 \pm 20.8$ & $958.6 \pm 10.1$ & $63.6 \pm 0.6$ & $<0.5$ \\
\hline $\mathrm{B}^{\#}$ & $1499.9 \pm 44.2$ & $606.9 \pm 26.5$ & $282.2 \pm 2.9$ & $40.8 \pm 2.2$ \\
\hline C & $1056.0 \pm 140.0$ & - & $1041.3 \pm 141.6$ & - \\
\hline D & $905.4 \pm 39.4$ & $885.6 \pm 46.5$ & $62.5 \pm 2.5$ & $<0.5$ \\
\hline$E$ & $1092.7 \pm 54.2$ & - & $1042.9 \pm 37.9$ & - \\
\hline $\mathrm{F}^{s}$ & $1045.1 \pm 43.1$ & $710.7 \pm 40.2$ & $336.9 \pm 12.9$ & $<0.5$ \\
\hline $\mathrm{G}^{\$ S}$ & $1019.3 \pm 8.6$ & $727.5 \pm 21.3$ & $321.4 \pm 13.7$ & $<0.5$ \\
\hline $\mathrm{H}$ & $8.8 \pm 0.2$ & - & $10.6 \pm 0.6$ & - \\
\hline I & $14.7 \pm 1.1$ & - & $10.2 \pm 1.8$ & - \\
\hline
\end{tabular}


Table 4. Concentrations of total fluoride (TF), total soluble fluoride (TSF as ion MFP and ion F) and the percentage of insoluble fluoride found after accelerated aging simulating one year of natural aging. Data are expressed as the mean \pm SD. Samples were analyzed in triplicate and the representative of the two independent experiments is shown.

\begin{tabular}{lcccc}
\hline \multirow{2}{*}{ Dentifrices } & $\begin{array}{c}\text { Total Fluoride } \\
\text { (TF) }\end{array}$ & \multicolumn{2}{c}{ Total soluble fluoride (TSF) } & \% Insoluble F \\
\cline { 3 - 5 } A & $981.9 \pm 9.6$ & $967.8 \pm 14.2$ & $66.0 \pm 2.4$ & $<0.5$ \\
\hline B & $1582.5 \pm 22.0$ & $540.1 \pm 15.5$ & $432.5 \pm 7.9$ & $38.5 \pm 0.9$ \\
\hline C & $932.1 \pm 22.6$ & - & $924.8 \pm 9.5$ & - \\
\hline D & $967.1 \pm 63.6$ & $854.1 \pm 89.6$ & $111.8 \pm 8.3$ & $<0.5$ \\
\hline E & $993.6 \pm 17.3$ & - & $1023.4 \pm 2.3$ & - \\
\hline F & $906.7 \pm 4.1$ & $458.5 \pm 23.2$ & $397.2 \pm 12.1$ & $5.6 \pm 1.7$ \\
\hline G & $1094.1 \pm 6.5$ & $502.8 \pm 4.1$ & $483.0 \pm 14.9$ & $9.9 \pm 0.6$ \\
\hline$H$ & $8.8 \pm 0.3$ & - & $10.3 \pm 0.8$ & - \\
\hline I & $9.7 \pm 0.7$ & - & $8.9 \pm 0.5$ & - \\
\hline
\end{tabular}

Table 5. Concentrations of total fluoride (TF), total soluble fluoride (TSF as ion MFP and ion F) and the percentage of insoluble fluoride found close to expiration. Data are expressed as the mean \pm SD. Samples were analyzed in triplicate and the representative of the two independent experiments is shown.

\begin{tabular}{lcccc}
\hline \multirow{2}{*}{ Dentifrices } & $\begin{array}{c}\text { Total Fluoride } \\
\text { (TF) }\end{array}$ & \multicolumn{2}{c}{ Total soluble fluoride (TSF) } & \% Insoluble F \\
\cline { 3 - 4 } & $1450.8 \pm 23.1$ & $335.1 \pm 39.4$ & $441.2 \pm 0.8$ & $44.9 \pm 1.1$ \\
\hline B & $926.9 \pm 5.2$ & - & $946.1 \pm 9.8$ & - \\
\hline E & $1010.4 \pm 9.4$ & - & $1063.4 \pm 11.5$ & - \\
\hline F & $710.6 \pm 0.9$ & $218.5 \pm 3.7$ & $403.4 \pm 0.6$ & $12.5 \pm 0.7$ \\
\hline G & $814.4 \pm 2.3$ & $265.8 \pm 1.9$ & $465.4 \pm 2.7$ & $10.3 \pm 0.4$ \\
\hline
\end{tabular}

ing MFP-based formulations had changes in their composition. When considering the concentration of soluble fluoride in the form of MFP, B, F and G showed a reduction of $20.9 \%, 43 \%$ and $6.9 \%$, respectively (Table 4 ). The calcium-containing, locally manufactured MFP-based formulations showed changes in the ionic concentrations as well. The concentration of ionic fluoride in B, F and $\mathrm{G}$ had increased from 244 to $433 \mathrm{ppm} F$, 179 to 397 ppm F and 233 to 483 ppm F, respectively. This increase caused a concomitant increase in insoluble fluoride in $\mathrm{B}, \mathrm{F}$ and $\mathrm{G}$ from $22.4 \%$ to $38.5 \%, 0.5 \%$ to 5.6 and $0.5 \%$ to $9.9 \%$, respectively. The soluble fluoride concentrations available after accelerated aging in $\mathrm{B}, \mathrm{F}$ and $\mathrm{G}$ ranged from $900-1000$ ppm $\mathrm{F}, 800-900 \mathrm{ppm} F$ and 900-1000 ppm F, respectively (Table 4).

With regard to the fluoride concentrations close to expiry dates (at the end of two years), NaF-based formulations ( $C$ and $E$ ) did not change markedly (Table 5). The same was observed with the MFP-based formulations ( $A$ and D) that did not contain calcium but contained hydrated silica (data not shown). However, all the calcium-containing MFP-based formulations ( $B, F$ and $G$ ) showed reduced total and soluble fluoride concentrations with an increased concentration of insoluble fluoride levels (Table 5). 


\section{Discussion}

Fluoride plays a vital role in the reduction of the prevalence of dental caries in the developed as well as developing countries. Tooth brushing with a fluoride toothpaste is considered the most important public measure to be taken to reduce the caries prevalence and moreover, it has become the easiest and the most convenient method of delivering fluoride. Fluoride in the form of a toothpaste exerts a local effect on teeth and prevents the solubility of minerals from the enamel and causes remineralization due to its availability at the site of action.

In the current study, we have used nine brands of toothpaste to check the fluoride levels at the time of acquisition, after aging and close to their expiry date to see the levels of fluoride. Two local herbal brands which were being advertized as not having fluoride were used as negative controls which reflected well in our study (Table 2). Here in this present study, we have used an established method of fluoride analysis based on the ion-selective electrode method which is considered the gold standard method in fluoride analysis ${ }^{16}$. Out of the seven fluoridated toothpastes, five contained fluoride as MFP, whereas two imported formulations contained NaF (Table1). Chemical compatibility of formulations in toothpastes is vital for the anti-caries effect. In our sample, $\mathrm{F}$, which is the active ingredient in a toothpaste, was available as $\mathrm{F}$ in inorganic salt, i.e. $\mathrm{NaF}$ or as MFP. Because $\mathrm{F}$ is available with phosphate $\left(\mathrm{PO}^{3}\right)$ in $\operatorname{MFP}\left(\mathrm{Na}_{2} \mathrm{FPO}_{3}\right), \mathrm{MFP}$ is stable in the presence of $\mathrm{CaCO}_{3}{ }^{(4,7)}$. However, due to the hydrolysis of $\mathrm{PO}_{3} \mathrm{~F}^{2-}$, the free $\mathrm{F}$ - ion binds to $\mathrm{Ca}^{2+}$ forming insoluble $\mathrm{CaF}_{2}$, which we observed in formulation $\mathrm{B}$ which had an insoluble $\mathrm{F}$ concentration of $22.4 \%$ even at the time of acquisition (Table 2).

According to the best evidence available ${ }^{8,17}$, at least a minimum of $1000 \mathrm{ppm} F$ should be available in a dentifrice as TSF but only two formulations were in accordance with the recommended amounts (Table 2). Nevertheless, all the fluoridated toothpastes contained at least 900 ppm F which may be considered acceptable, given the fact that local standards in Sri Lanka permit concentrations below 1000 ppm F. However, the standards do not specify whether F should be available as TF or TSF. In a similar study done in Malaysia it has been reported that only two toothpastes contained $F$ which was about 1000 ppm $F$ whereas the rest of the toothpastes contained $F$ in the range of 20-470 ppm F with one toothpaste containing 870 ppm $\mathrm{F}^{18}$. In contrast to the present study, the majority of the toothpastes tested in the aforementioned study had very low levels of $F$ which were inadequate to exert the anti-caries effect of F. Even Japan being an industrialized country, prohibits the use of fluoride more than $1000 \mathrm{ppm} F$ by their legislation, accounting for less than $1000 \mathrm{ppm} \mathrm{F}$ in most consumed dentifrices ${ }^{10}$. A recent report from India also revealed that out of 12 toothpastes they had tested only two had standard concentrations of 1000 ppm F while the rest had TSF concentrations between 825-982 ppm $\mathrm{F}^{19}$. On the other hand, developed countries like USA, Australia and Europe specify that F- in NaF or MFP should not be less than $60 \%$ of total fluoride, which should be maintained throughout the shelf life.

Accelerated aging did not affect the non-fluoridated and NaF-based formulations (Table 4). Minor differences observed in these formulations probably were due to analytical variations. In the case of formulation $B$, the ionic $F$ concentration increased from 244 to 433 ppm F, suggesting that further reactions of $F$ with the calcium in the abra- 
sive would occur, increasing the insoluble F (inactive F) with time (Table 4). The same pattern was observed with formulations $F$ and $G$ as well. Although aging affected the calcium containing MFP-based formulations ( $B, F$ and $G$ ) causing a reduction in the levels of soluble $F$, the soluble $F$ concentration was still close to $900 \mathrm{ppm} F$ which was within the accepted levels in Sri Lanka. In a similar study done in Japan using $\mathrm{MFP} / \mathrm{CaCO}_{3}$-based formulations which had TSF concentrations below 1000 ppm F at the time of acquisition, it has been shown that aging of toothpastes after one year causes a reduction in TSF by $15 \%$ with a concomitant increase in insoluble fluoride ${ }^{10}$.

At the time of expiration, natural aging did not affect the NaF-based formulations ( $C$ and $E$ ) as well as MFP-based formulations with hydrated silica, suggesting that $\mathrm{SiO}_{2}$ allows all the $\mathrm{F}$ to remain in the soluble form throughout the shelf life. This was also observed in the aforementioned Japanese study which showed that MFP-based formulations containing silica as an abrasive did not change with aging ${ }^{10}$. Our data clearly shows that not all the toothpastes were able to maintain recommended levels of soluble $F$ until their expiration date to exert the maximum anti-caries effect. On the other hand, the time period that toothpastes stay on the shelves in the stores is less than the evaluated period, i.e. one year and 2 years, suggesting that if used within a year, the toothpastes might contain $\mathrm{F}$ levels of Sri Lankan standards.

By the time of the expiration date, formulation B had $44 \%$ of insoluble $F$ which suggests that the reactions of $F$ with calcium had occurred in the first few months (within 6 months) of manufacturing the toothpaste. Though $F$ and $G$ formulations showed the same pattern of an increase in insoluble $F$, these formulations seemed to be more stable than B as only $10 \%$ of total soluble $F$ was lost by the time of the expiration date.

It has been shown that high temperatures can enhance the hydrolysis of MFP, causing more insoluble fluoride to be produced ${ }^{19,20}$. Given the fact that Sri Lanka is a tropical country having high temperatures in certain parts of the country, it can be assumed that further hydrolysis of MFP-based formulations can occur in these areas, which requires further research. Even though we cannot come to a conclusive decision due to certain limitations of the study, it is apparent that the TSF concentration in the formulations does not meet the desirable minimum values ${ }^{8,17}$. Further studies are necessary with large sample numbers with different batches to check whether the concentrations fluctuate between each batch of a particular formulation and in different climates as well.

In the light of aforementioned data, it is apparent that all the MFP-based formulations available in Sri Lanka contain TSF levels close to the concentrations accepted by Sri Lankan standards. However, it has been well established by a systematic review that a minimum of 1000 ppm F or above should be available for the anti-caries effect. Therefore, authorities in Sri Lanka should pay heed to this evidence-based information and act accordingly, given the fact that the caries prevalence in Sri Lanka is not declining despite the measures the government has taken.

In conclusion, most of the dentifrices marketed in Sri Lanka contain TSF less than $1000 \mathrm{ppm}$ F. Furthermore, MFP/CaCO${ }_{3}$-based formulations show reduced levels of TSF with aging and an increase in inactive $\mathrm{CaF}_{2}$ in toothpastes. Hence, it is recommended to check the manufacturing date before buying a toothpaste and to refrain 
from buying a toothpaste closer to its expiry date if it contains $\mathrm{MFP} / \mathrm{CaCO}_{3}$. The authorities should pay heed to the best evidence and recommendations should be made accordingly to have a TSF level of $1000 \mathrm{ppm} \mathrm{F}$ and maintain it throughout the lifespan of a toothpaste.

\section{Acknowledgement}

The research was supported by the University Research Grant RG/ 2014/15/D. The authors wish to acknowledge Ms. Harshini Pethiyagoda of the English Language Teaching Unit (ELTU) of the Faculty of Sciences, University of Peradeniya, Sri Lanka for professionally editing the language of the manuscript.

\section{References}

1. Cury JA, Tenuta LMA. How to maintain a cariostatic fluoride concentration in the oral environment. Adv Dent Res. 2008 Jul 1;20(1):13-6.

2. Shellis RP, Duckworth RM. Studies on the cariostatic mechanisms of fluoride. Int Dent J. 1994 Jun;44(3 Suppl 1):263-73.

3. Brattall D, Hansel-Peterson G, Sunberg H. Reason for the caries decline: What do the experts believe? Eur J Oral Sci. 1996 Aug;104(4 ( Pt 2)):416-22; discussion 423-5, 430-2.

4. Cury JA, Tenuta LM, Ribeiro CC, PaesLeme AF. The importance of fluoride dentifrices to the current dental caries prevalence in Brazil. Braz Dent J. 2004;15(3):167-74.

5. Birkeland JM, Haugejorden O, von der Fehr FR. Some factors associated with the caries decline among Norwegian children and adolescents: age-specific and cohort analyses. Caries Res. 2000;34(2):109-16. doi: 10.1159/000016577.

6. Tenuta LM, Cury JA. Laboratory and human studies to estimate anticaries efficacy of fluoride toothpastes. Monogr Oral Sci. 2013;23:108-24. doi: 10.1159/000350479.

7. Ricomini Filho AP, Tenuta LMA, Fernandes FS, Calvo AFB, Kusano SC, Cury JA. Fluoride concentration in the top-selling Brazilian toothpastes purchased at different regions. Braz Dent J 2012;23(1):45-8.

8. Walsh T, Worthington HV, Glenny AM, Appelbe P, Marinho VC, Shi X. Fluoride toothpastes of different concentrations for preventing dental caries in children and adolescents. Cochrane Database Syst Rev. 2010 Jan 20;(1):CD007868. doi: 10.1002/14651858.CD007868.pub2.

9. Freitas JF. Fluoride stability in toothpastes. Aust Dent J. 1984 Feb;29(1):30-5.

10. Hashizume LN, Lima YB, Kawaguchi Y, Cury JA. Fluoride availability and stability of Japanese dentifrices. J Oral Sci. 2003 Dec;45(4):193-9.

11. Matias JB, Azevedo CS, do Vale HF, Rebelo MA, Cohen-Carneiro F. Fluoride stability in dentifrices stored in schools in a town of northern Brazil. Braz Oral Res. 2015;29(1):S1806-83242015000100304. doi: 10.1590/1807-3107BOR-2015.vol29.0121.

12. Sri Lanka. Ministry of Health and Nutrition. National Oral Health Survey 1994-1995.

13. Sri Lanka. Ministry of Health and Nutrition. National Oral Health Survey 2002-2003.

14. Cury JA, Oliveira MJ, Martins CC, Tenuta LM, Paiva SM. Available fluoride in toothpastes used by Brazilian children. Braz Dent J. 2010;21(5):396-400.

15. Tabchoury CPM, Cury JA. [Study of toothpaste aging conditions to predict fluoride behavior in environment conditions]. Rev Bras Farm. 1994;75(3):67-71. Portuguese. 
16. Martínez-Mier EA, Cury JA, Heilman JR, Katz BP, Levy SM, Li Y, et al. Development of gold standard ion-selective electrode-based methods for fluoride analysis. 2011;45(1):3-12. doi: $10.1159 / 000321657$.

17. dos Santos AP, Nadanovsky P, de Oliveira BH. A systematic review and meta-analysis of the effects of fluoride toothpastes on the prevention of dental caries in the primary dentition of preschool children. Evid Based Dent. 2014; 1: 5. Evid Based Dent. 2014 Sep;15(3):67. doi: 10.1038/sj.ebd.6401035.

18. Abdul-Kadir R, Abdol-Latif L. Fluoride levels in dentifrices. Ann Dent Univ Malaya. 1998;5:2-5.

19. Sebastian ST and Siddanna S. Total and free fluoride concentration in various brands of toothpastes marketed in India. J Clin Diagn Res. 2015 Oct;9(10):ZC09-12. doi: 10.7860/JCDR/2015/13382.6578.

20. Conde NC, Rebelo MA, Cury JA. Evaluation of the fluoride stability of dentifrices sold in Manaus, AM, Brazil. Pesqui Odontol Bras. 2003 Jul-Sep;17(3):247-53. 